\title{
The Phoenix radio survey: The angular correlation function
}

\author{
A. Georgakakis ${ }^{1,2}$, B. Mobasher ${ }^{2}$, L. Cram $^{3}$, A. Hopkins ${ }^{4}$, and M. Rowan-Robinson ${ }^{2}$ \\ 1 School of Physics and Astronomy, University of Birmingham, Edgbaston B15 2TT, UK \\ 2 Astrophysics Group, Blackett Laboratory, Imperial College, Prince Consort Rd, London SW7 2BZ, UK \\ 3 Astrophysics Department, School of Physics, University of Sydney, NSW 2006, Australia \\ 4 Department of Physics and Astronomy, University of Pittsburgh, 3941 O'Hara Street, PA 15260, U.S.A.
}

Received February 11; accepted October 18, 1999

\begin{abstract}
Using the Phoenix radio survey, a homogeneous survey selected at $1.4 \mathrm{GHz}$ and covering an area of $\approx 3 \mathrm{deg}^{2}$, we analyse the clustering of the sub-mJy radio population using angular correlation function analysis. Extensive simulations are carried out to investigate the significance of the estimated angular correlation amplitudes. Our analysis show that for the $S_{1.4}>0.5 \mathrm{mJy}$ sub-samples the radio source distribution is anisotropic at the $2 \sigma$ significance level. Additionally, we estimate upper limits for the angular correlation amplitudes that, despite the large uncertainties, are in good agreement with the amplitude estimates for sources brighter than $1 \mathrm{mJy}$, detected in the FIRST radio survey (Cress et al. 1997). Adopting a radio luminosity function and assuming an evolving spatial correlation function of the form $\xi(r)=$ $\left(r / r_{0}\right)^{-\gamma}(1+z)^{-(3+\epsilon)}$, with the evolution parametrised by $\epsilon$, we find an upper limit for the angular correlation length $r_{0} \approx 9 h^{-1} \mathrm{Mpc}$ for $S_{1.4}>0.5 \mathrm{mJy}$ and $\gamma=2.1$. This agrees well with the value $r_{0} \approx 6-8 h^{-1} \mathrm{Mpc}$ estimated from the FIRST radio survey for sources brighter than $1 \mathrm{mJy}$. Additionally, we quantify the characteristics, in terms of areal coverage and limiting flux density, of future deep radio surveys to yield a significant correlation amplitude detection and to explore possible changes of the correlation amplitude with flux density.
\end{abstract}

Key words: galaxies: general — galaxies: structure cosmology: large scale structure of universe — radio continuum: galaxies

\section{Introduction}

The spatial and angular correlation functions, $\xi(r)$ and $w(\theta)$ respectively, have been extensively used to quantify the clustering of galaxies. The former has the important property of being the Fourier conjugate of the power spectrum, $P(k)$, of the galaxy distribution (e.g. Peacock $\&$ Nicholson 1991). The latter is the two dimensional projection of $\xi(r)$ on the sky and is related to it by the Limber equation (Limber 1953; Phillipps et al. 1978). Although $w(\theta)$ conveys less information on galaxy clustering than $\xi(r)$ due to its integral nature, nevertheless it is a powerful probe of the large scale distribution of galaxies, especially at faint magnitudes where redshift information is difficult to obtain.

Wide-area surveys at optical wavelengths have provided information on the large-scale structure at redshifts $z \approx 0.1$ (Loveday et al. 1995; Maddox et al. 1990b). Additionally, deep optical and near-infrared samples over smaller areas (Villumsen et al. 1997; Hudon et al. 1996; Roche et al. 1998a,b; Carlberg et al. 1997) give useful information on the large-scale distribution of galaxies at higher redshifts $(z \leq 1.0)$. There is a general consensus that at optical wavelengths, $w(\theta)$ is a power law of the form $w(\theta)=A_{w} \theta^{-(\gamma-1)}$, with $\gamma \approx 1.8$ (Maddox et al. 1990b). The amplitude, $A_{w}$, decreases with increasing depth of the survey, while there is also evidence that the slope, $\gamma$, flattens at faint magnitudes (Infante $\&$ Pritchet 1995). The spatial correlation function has the form $\xi(r)=\left(r / r_{0}\right)^{-\gamma}$, where the correlation length, $r_{0}$, is found to be $r_{0}=5.4 h^{-1} \mathrm{Mpc}$ (Davis \& Peebles 1983). However, it has been demonstrated that early-type galaxies are more clustered (higher values of $r_{0}$ ) than late types and that lower luminosity galaxies are a factor of $\approx 2$ less clustered than their brighter counterparts of the same Hubble type (Loveday et al. 1995). There is also evidence that $r_{0}$ decreases with increasing depth of the survey (Infante \& Pritchet 1995), due to the presence of a population of weakly clustered faint galaxies.

Radio surveys, unlike the optical ones, are not affected by galactic dust extinction and mainly consist of high redshift objects $(\bar{z} \approx 1)$. Therefore, they sample much larger volumes than optically selected samples, albeit with sparser coverage, providing the opportunity to study the clustering of matter at much larger 
physical scales. However, the broad luminosity function of extragalactic radio sources (Condon 1984; Dunlop \& Peacock 1990) implies that a flux density-limited sample spans a wide range of redshifts. The projection on the sky of all detected objects results in a distribution close to random, smearing out any information on the large-scale structure. This is supported by the fact that no signal has been detected in the angular (2-D) correlation analysis of bright radio galaxies (Webster 1976; Masson 1979). However, Shaver \& Pierre (1989) found evidence of anisotropic distribution of radio sources towards the supergalactic plane extending out to at least $z \approx 0.02$. More recently, a clustering length of $r_{0}=11 h^{-1} \mathrm{Mpc}$ was estimated at $1.4 \mathrm{GHz}$ from the spatial (3-D) correlation analysis of radio sources having flux densities $>0.5 \mathrm{Jy}$ and redshifts in the range $0.01<z<0.1$ (Peacock \& Nicholson 1991).

Cress et al. (1996) have suggested that at lower flux densities, projection effects may become less significant and hence, a 2-D correlation analysis can be applied successfully to investigate the clustering of radio galaxies. Indeed, a non-zero amplitude has been found for the angular correlation function of radio sources with $S_{4.85 \mathrm{GHz}}>50 \mathrm{mJy}$ in both the Green Bank (northern hemisphere) and the Parkes-MIT-NRAO (southern hemisphere) 4.85 GHz sky surveys (Loan et al. 1997; Kooiman et al. 1995). More recently, using the FIRST radio survey (1.4 GHz; Becker et al. 1995), with a uniform sensitivity of $1 \mathrm{mJy}$ over an area of $1500 \mathrm{deg}^{2}$, a non-zero and clearly significant amplitude for the angular correlation function is estimated (Cress et al. 1996). Adopting the radio luminosity functions (RLF) determined independently by Condon (1984) and Dunlop \& Peacock (1990), Cress et al. (1997) inferred a clustering length of $r_{0}=6-8 h^{-1} \mathrm{Mpc}$ for $S_{1.4}>1$ mJy radio sources.

The recent large-area radio surveys described above, have provided information on the two-dimensional projected distribution of relatively bright $\left(S_{1.4} \geq 1 \mathrm{mJy}\right)$ radio sources, dominated by bright ellipticals and AGNs. However, there is still limited information on the clustering properties of the faint (sub-mJy) radio population. At flux densities below few mJy there is evidence for the appearance of a new population of faint radio sources, likely to comprise a large fraction of starbursts (Benn et al. 1993; Georgakakis et al. 1999). In particular, the radio luminosity function models developed by Condon (1984) predict that the surface density of starbursts increases from $10 \%$ of the radio population at $S_{1.4} \approx 1 \mathrm{mJy}$ to over $30 \%$ at $S_{1.4} \approx 0.4 \mathrm{mJy}$. Moreover, studies of the spatial distribution of starbursts, selected at optical and infrared wavelengths, show that these objects are expected to have different clustering properties (Davis \& Geller 1976; Giovanelli et al. 1986; Saunders et al. 1992; Loveday et al. 1995) compared to those of early-type galaxies, with which bright radio sources are often associated. Therefore, study of the angular correlation function at sub-mJy flux density levels has the potential to reveal differences in the clustering properties of the faint radio population.

Recently, Benn \& Wall (1995) demonstrated that the isotropy (or anisotropy) in the radio source counts in different fields of similar geometry and sensitivity can be used to set limits on the scale of the largest cellular structures in the Universe. However, they argued that at submJy and $\mu$ Jy flux densities, such a study is hampered by the small number of comparable surveys and by the small number of sources detected in each field. This is also supported by Windhorst et al. (1990), who reviewed the field-to-field variations in the radio source counts of small area radio surveys $\left(\leq 1 \mathrm{deg}^{2}\right)$. They concluded that the differences, although exceeding the random distribution expectation, are due to statistical fluctuations, rather than of cosmic nature. Nevertheless, a non-uniform angular correlation function was estimated by Oort (1987a) for the radio sources detected in the deep $\left(S_{1.4}>0.1 \mathrm{mJy}\right)$ Lynx fields (Oort 1987b). More recently, Richards (1999) also reported the detection of clustering signal for radio sources brighter than $40 \mu \mathrm{Jy}$, detected within a 40 arcmin diameter radio survey $(1.4 \mathrm{GHz})$ centred on the HDF.

The Phoenix radio $(1.4 \mathrm{GHz})$ survey, covering an area of $3.14 \mathrm{deg}^{2}$, larger than any other survey at a similar flux density limit $\left(S_{1.4}=0.4 \mathrm{mJy}\right)$ and reaching surface densities $\approx 6.610^{5}$ sources $\mathrm{sr}^{-1}$, provides a unique opportunity to study the clustering properties of the faint radio population. In this paper the angular correlation function of the radio sources detected in the Phoenix field is estimated. Section 2 gives a brief description of the observations. The method for calculating $w(\theta)$ is outlined in Sect. 3. In Sect. 4 we estimate the correlation function of the radio sample. Section 5 presents the simulations carried out to investigate the significance of our results, while in Sect. 6 the correlation function amplitudes are compared with $3-\mathrm{D}$ clustering models. The results from the radio correlation analysis are discussed in Sect. 7. Finally, we summarise our conclusions in Sect. 8.

\section{Radio observations}

The Phoenix survey has been performed at $1.4 \mathrm{GHz}$ using the 6A configuration of the Australia Telescope Compact Array (ATCA). It is a mosaic of 30 pointing centres covering a $2^{\circ}$ diameter field centred at $\mathrm{RA}(2000)=$ $01^{\mathrm{h}} 14^{\mathrm{m}} 12^{\mathrm{s}} .16 ;$ Dec. $(2000)=-45^{\circ} 44^{\prime} 8^{\prime \prime} .0$. The final synthesised beam size is $\approx 8$ arcsec.

Details of the observation, data reduction, source detection and catalogue generation are presented in Hopkins et al. (1998). A source is included in the sample if its peak flux density is $4 \sigma$ above the local $\mathrm{rms}$ and if it survives visual inspection. There are two sources of incompleteness in the catalogue, as with any peak flux density limited sample. The first is a result of the attenuation of the primary beam sensitivity away from a field centre. This effect 
has been minimised in the Phoenix survey by the mosaicing strategy used. The second is the fact that extended objects with a total flux density above the survey limit can be missed by a source detection algorithm which initially detects candidates based on their peak flux densities. These effects have been described in Hopkins et al. (1998). The catalogue is estimated to be $50 \%$ complete to $0.3 \mathrm{mJy}$, and $80 \%$ complete to $0.4 \mathrm{mJy}$. Therefore to minimise the effect of incompleteness when performing the correlation analysis, we restrict ourselves to a subsample containing all sources brighter than $0.4 \mathrm{mJy}$.

\section{Two point correlation function}

The two-point angular correlation function, $w(\theta)$, is defined as the joint probability $\delta P$ of finding sources within the solid angle elements $\delta \Omega_{1}$ and $\delta \Omega_{2}$, separated by an angle $\theta$, in the form

$\delta P=N^{2}(1+w(\theta)) \delta \Omega_{1} \delta \Omega_{2}$,

where $N$ is the mean surface density of galaxies. For a random distribution of sources $w(\theta)=0$. Therefore, the angular correlation function provides a measure of galaxy density excess over that expected for a random distribution. Various methods for estimating $w(\theta)$ have been introduced, as discussed by Infante (1994). In the present study, a source is taken as the "centre" and the number of pairs within annular rings is counted. To account for the edge effects, Monte Carlo techniques are used by placing random points within the area of the survey. The most commonly used estimators in this procedure have the form

$w=\frac{D D}{R R}-1$, or

$w=\frac{D D}{D R}-1$

where DD and DR are respectively the number of sources and random points computed at separations $\theta$ and $\theta+\mathrm{d} \theta$ from a given galaxy. Similarly, RR is the number of random points within the angular interval $\theta$ to $\theta+\mathrm{d} \theta$ from a given random point. Infante (1994) and Hewett (1982) emphasised the importance of correcting for any spurious cross correlation between the random and the galaxy catalogues when using the above estimators, arising partly because of the anisotropic and inhomogeneous galaxy distribution relative to the field boundaries. The corrected form of the estimator is then

$w=\frac{D D}{D R}-\frac{R D}{R R}$

where RD is the number of random-data pairs, taking the random points as centres (Infante 1994). Landy \& Szalay (1993, hereafter LS) introduced the estimator

$w=\frac{D D-2 D R+R R}{R R}$.
The advantages of this estimator is that it minimises the variance of $w(\theta)$ and also reduces the edge effects from which both the estimators (3) and (4) suffer. Moreover, it has similar properties (Landy \& Szalay 1993) to that introduced by Hamilton (1993)

$w=\frac{D D R R}{D R D R}-1$.

In the present study the correlation function has been calculated using estimators (4), (5) and (6). The results agree within the respective uncertainties. Therefore, only the results from the LS estimator are presented.

For a given radio galaxy sample, a total of 100 random catalogues were generated, each having the same number of points as the original data set. The random sets were cross-correlated with the galaxy catalogue, giving an average value for $w(\theta)$ at each angular separation. In producing random sets of points, we take into account variations in sensitivity, which might affect the correlation function estimate. The flux density threshold for detection depends on the local rms noise which varies across the survey area. Since the random fields are expected to have the same sources of bias as the data (i.e. the simulated catalogues must have the same selection effects as the real catalogue), the rms noise map, with a resolution of $\approx 10$ arcsec, is used to discard simulated points in noisy areas. This is accomplished, to the first approximation, by assigning a flux density to random points using the Windhorst et al. (1985) $\log N-\log S$ distribution, following the method of Cress et al. (1996). If the flux density of the random point is less than 4 times the local rms noise, the point is excluded from the random data set. However, producing random points with a uniform distribution over the observed area does not change our final result.

The uncertainty in $w(\theta)$ is determined using both Poisson statistics and 50 bootstrap resamples of the data (Ling et al. 1986). For the latter method, simulated datasets were generated by sampling $\mathrm{N}$ points with replacement from the true data-set of $\mathrm{N}$ points. The correlation function is then calculated for each of the bootstrap samples, following the same procedure as that with the original data-set. The standard deviation around the mean is then used to estimate the uncertainty in the correlation function.

Although the LS estimator used here is shown to have Poissonian variance for uncorrelated points (Landy \& Szalay 1993), it does not necessarily behave this way for correlated data. The bootstrap method is believed to give a more representative estimate of the uncertainty associated with $w(\theta)$. However, Fisher et al. (1994) carried out a detailed study of the biases affecting the bootstrap errors (e.g. cosmic variance, sparse sampling by galaxies of the underlying density distribution) and concluded that overall the bootstrap uncertainties overestimate the true errors. Nevertheless, the bootstrap resampling is a general method for assessing the accuracy of the angular correlation function estimator and it will be used here to calculate 
Table 1. Number of sources in each flux density-limited subsample

\begin{tabular}{cc}
\hline Flux density (mJy) & Number of Sources \\
\hline$>0.4$ & 634 \\
$>0.5$ & 529 \\
$>0.6$ & 454 \\
$>0.7$ & 391 \\
$>0.9$ & 316 \\
{$[0.4,0.9]$} & 318 \\
\hline
\end{tabular}

the formal errors. The uncertainty associated with $w(\theta)$, estimated by the bootstrap resampling technique, is found to be about three times larger than the Poisson estimate.

There is expected be a significant number of physical double sources in a radio survey with the angular resolution of the Phoenix survey $\left(\approx 8^{\prime \prime}\right)$. In any study of the clustering of radio galaxies via correlation analysis, these should not be considered as two sources since both components are formed in the same galaxy. To identify groups of sources that are likely to be sub-components of a single source we have employed a percolation technique where all sources within a given radius are replaced by a single source at an appropriate "centroid" (Cress et al. 1996; Magliocchetti et al. 1998). Following the method developed by Magliocchetti et al. (1998), we vary the linklength in the percolation procedure according to the flux of each source. In that way, bright sources are combined, even if their angular separation is large, whereas faint sources are left as single objects. This technique is based on the $\theta-S$ relation for radio sources, which has been shown to follow $\theta \propto \sqrt{S}$ (Oort 1987a).

To define the relation between flux density and linklength, the angular separation of double sources versus their total flux is plotted in Fig. 1, out to a separation of 180 arcsec. Visual inspection has confirmed that the pairs on the left of Fig. 1 are predominantly sub-components of a single source. This is based on an assessment of the appearance of the object, including the disposition of the sources, the nature of any bridging radiation, and the appearance of source edges. Accordingly, we set the maximum link-length to be

$\theta_{\text {link }}=20 \times \sqrt{F_{\text {total }}}$,

where $F_{\text {total }}$ is the total flux of each group. This is shown by the dashed line in Fig. 1 and effectively removes the majority of visually identified doubles. Moreover, one can apply an additional criterion to identify genuine doubles, based on the relative flux densities of the sub-components (Magliocchetti et al. 1998). This is because lobes of a single radio source are expected to have correlated flux densities. Here, the groups of sources identified by the percolation technique are combined only if their fluxes differ by a factor of less than 4 . This procedure was repeated until no new groups were found. The final catalogue consists of 908

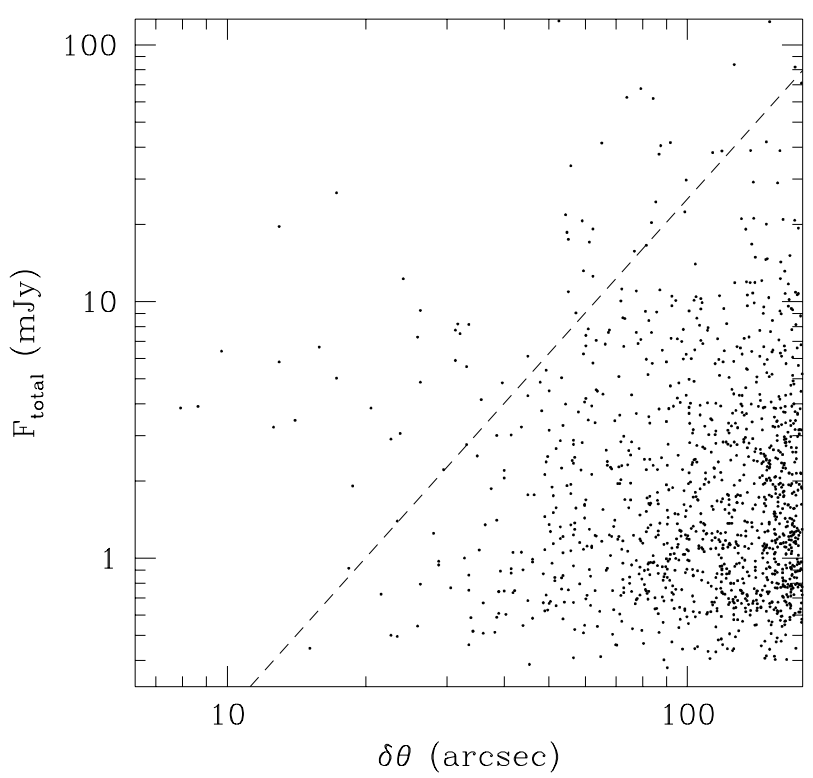

Fig. 1. Angular separation against total flux density of double sources. The dashed line represents the maximum link-length, for a given flux density, used in the percolation technique

objects to the limit of $0.1 \mathrm{mJy}$, with a total of 30 groups of sources being identified and replaced.

The source counts of the sample, normalised to the Euclidean slope, are plotted in Fig. 2 along with the radio counts at $1.4 \mathrm{GHz}$ taken from Windhorst et al. (1993). There is a drop in the number counts below $0.5 \mathrm{mJy}$, as our sample is affected by incompleteness. To quantify this, we firstly fit a straight line to the source counts of Windhorst et al. (1993) for flux densities fainter that 5 mJy (continuous line in Fig. 2). We then compare our number counts at a given flux density bin with those predicted by the fitted line. We conclude that our sample is $\approx 80 \%$ complete to $0.4 \mathrm{mJy}$. This is in agreement with the correction factors for incompleteness independently derived by Hopkins et al. (1998) accounting for both resolution effects and the attenuation of the beam away from the field centre. Therefore, to minimise the effect of incompleteness of the radio catalogue, when performing the correlation analysis, we restrict ourselves to a subsample containing all sources brighter than $0.4 \mathrm{mJy}$.

Finally, before fitting a power law to $w(\theta)$, we take into account a bias arising from the finite boundary of the sample. Since the angular correlation function is calculated within a region of solid angle $\Omega$, the background projected density of sources, at a given flux density limit $S_{\mathrm{o}}$, is effectively $N_{\mathrm{s}}\left(S>S_{\mathrm{o}}\right) / \Omega\left(\right.$ where $N_{\mathrm{s}}\left(S>S_{\mathrm{o}}\right)$ is the number of detected objects brighter than $S_{\mathrm{o}}$ ). However, this is an overestimation of the true underlying mean surface density, because of the positive correlation between galaxies in small separations, balanced by negative values of $w(\theta)$ at larger separations. This bias, known as the 


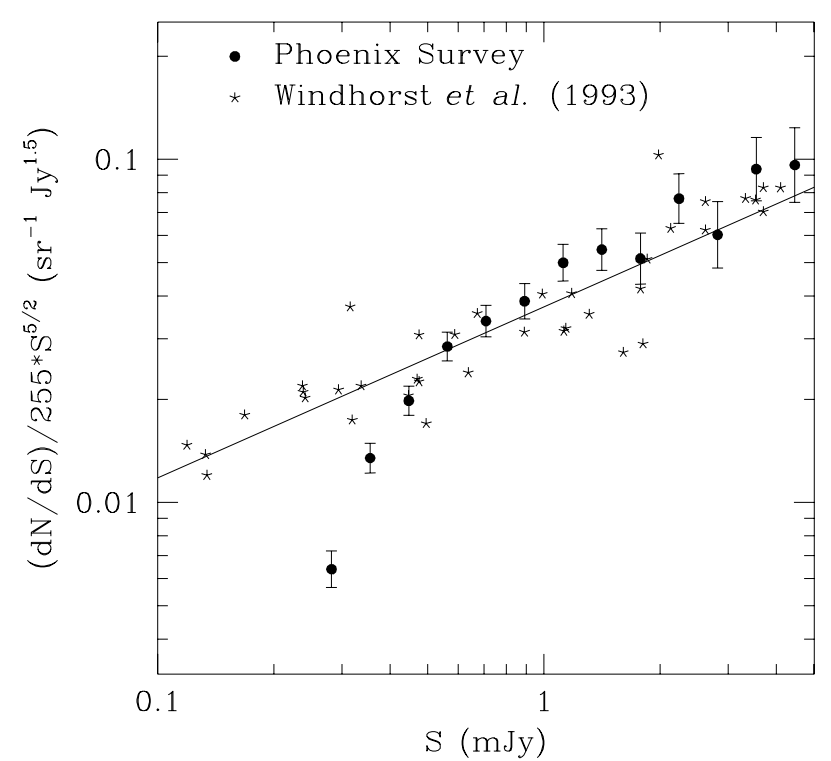

Fig. 2. Circles: source counts at $1.4 \mathrm{GHz}$ for the Phoenix Survey; Stars: source counts from Windhorst et al. (1993); Line: best fit to the Windhorst et al. (1993) source counts for flux densities less than $5 \mathrm{mJy}$

integral constraint, has the effect of reducing the amplitude of the correlation function by

$\omega_{\Omega}=\frac{1}{\Omega^{2}} \iint w(\theta) \mathrm{d} \Omega_{1} \mathrm{~d} \Omega_{2}$,

where $\Omega$ is the solid angle of the survey area. The angular constraint is estimated using Monte Carlo integration, assuming the correlation function to follow a power law $w(\theta)=A_{\omega} \times \theta^{-\delta}$ with $\delta=0.8$ or 1.1. We find $\omega_{\Omega}=1.46 A_{\omega}$ and $\omega_{\Omega}=1.89 A_{\omega}$ for $\delta=0.8$ and $\delta=1.1$ respectively, where $\theta$ is measured in degrees.

\section{Correlation function of the Phoenix radio survey}

We have extracted from the original catalogue four flux limited samples with flux density cutoffs at 0.4, 0.5, 0.6 and $0.7 \mathrm{mJy}$. Furthermore, to investigate changes in the clustering properties of radio sources with flux density, two independent sub-samples were considered, having the same number of objects and flux densities in the range $0.4<S_{1.4}<0.9 \mathrm{mJy}$ and $S_{1.4}>0.9 \mathrm{mJy}$. Table 1 lists the number of sources in each sub-sample. The correlation function $w(\theta)$ is calculated for each sub-sample for angular separations ranging from $0.03 \mathrm{deg}$ to $1.3 \mathrm{deg}$, within 9 equally separated logarithmic bins. The results are shown in Figs. 3 and 4.

The amplitude of the correlation function is estimated by fitting the following function to the observations

$w(\theta)=A_{w} \times \theta^{-\delta}-\omega_{\Omega}(\delta)$.

where the integral constraint is evaluated for the two different values of $\delta$ adopted in previous studies; $\delta=0.8$
Table 2. Angular correlation function amplitudes and the associated Poisson errors

\begin{tabular}{ccc}
\hline Flux Density $(\mathrm{mJy})$ & $A_{w}^{(\delta=1.1)}$ & $A_{w}^{(\delta=0.8)}$ \\
\hline$>0.4$ & $0.001 \pm 0.001$ & $0.003 \pm 0.003$ \\
$>0.5$ & $0.003 \pm 0.001$ & $0.006 \pm 0.003$ \\
$>0.6$ & $0.004 \pm 0.002$ & $0.008 \pm 0.004$ \\
$>0.7$ & $0.005 \pm 0.002$ & $0.010 \pm 0.004$ \\
$>0.9$ & $0.004 \pm 0.002$ & $0.009 \pm 0.005$ \\
{$[0.4,0.9]$} & $0.003 \pm 0.002$ & $0.007 \pm 0.005$ \\
\hline
\end{tabular}

(Peacock \& Nicholson 1991; Loan et al. 1997) and $\delta=1.1$ (Cress et al. 1996). The calculated amplitudes and the Poissonian uncertainties are listed in Table 2. Assuming Poisson errors, these amplitudes are non-zero at the $2 \sigma$ confidence level for all the sub-samples, except from those with $S_{1.4}>0.4 \mathrm{mJy}$ and $0.4<S_{1.4}<0.9 \mathrm{mJy}$. However, since the formal bootstrap errors are $\approx 3$ times larger than the Poisson expectation, the amplitudes derived here are treated as upper limits.

Additionally, there are two effects that might further increase the correlation amplitude errors: (i) the interdependence of the $w(\theta)$ measurements at different angles $\theta$ and (ii) the dependence of the uncertainty estimates on higher order correlations (skewness \& kurtosis) that are ignored in this study. However, Mo et al. (1992) demonstrated that the underestimation of the correlation amplitude uncertainty, $\delta A_{w}$, by these two effects is compensated by the overestimation of the $w(\theta)$ errors by the bootstrap resampling method.

Furthermore, because of the small solid angle of the Phoenix field, cosmic variance may affect the estimated correlation amplitudes. The effect of cosmic variance is studied in detail in the next section.

\section{Simulations}

Because of the relatively small Phoenix field size, one should be cautious about the interpretation of the correlation amplitude upper limits, since field-to-field fluctuations may affect our results. For example Oort (1987a) carried out the angular correlation function analysis using sub-mJy radio surveys smaller than Phoenix and found evidence for anisotropic source distribution in only some of them.

Firstly, to test the significance of our results we construct catalogues of randomly distributed points and calculate the correlation function from a region with the same geometry as the Phoenix field. In all trials, the estimated amplitudes are found to be consistent with zero within the Poisson standard deviation, $\sigma_{\text {Poisson }}$ (Fig. 5). Therefore, the amplitudes derived here (non-zero at the $2 \sigma_{\text {Poisson }}$ significance level), imply a non-uniform distribution of the faint radio population, albeit at the $2 \sigma$ confidence level. The exceptions are the $S_{1.4} \geq 0.4 \mathrm{mJy}$ 

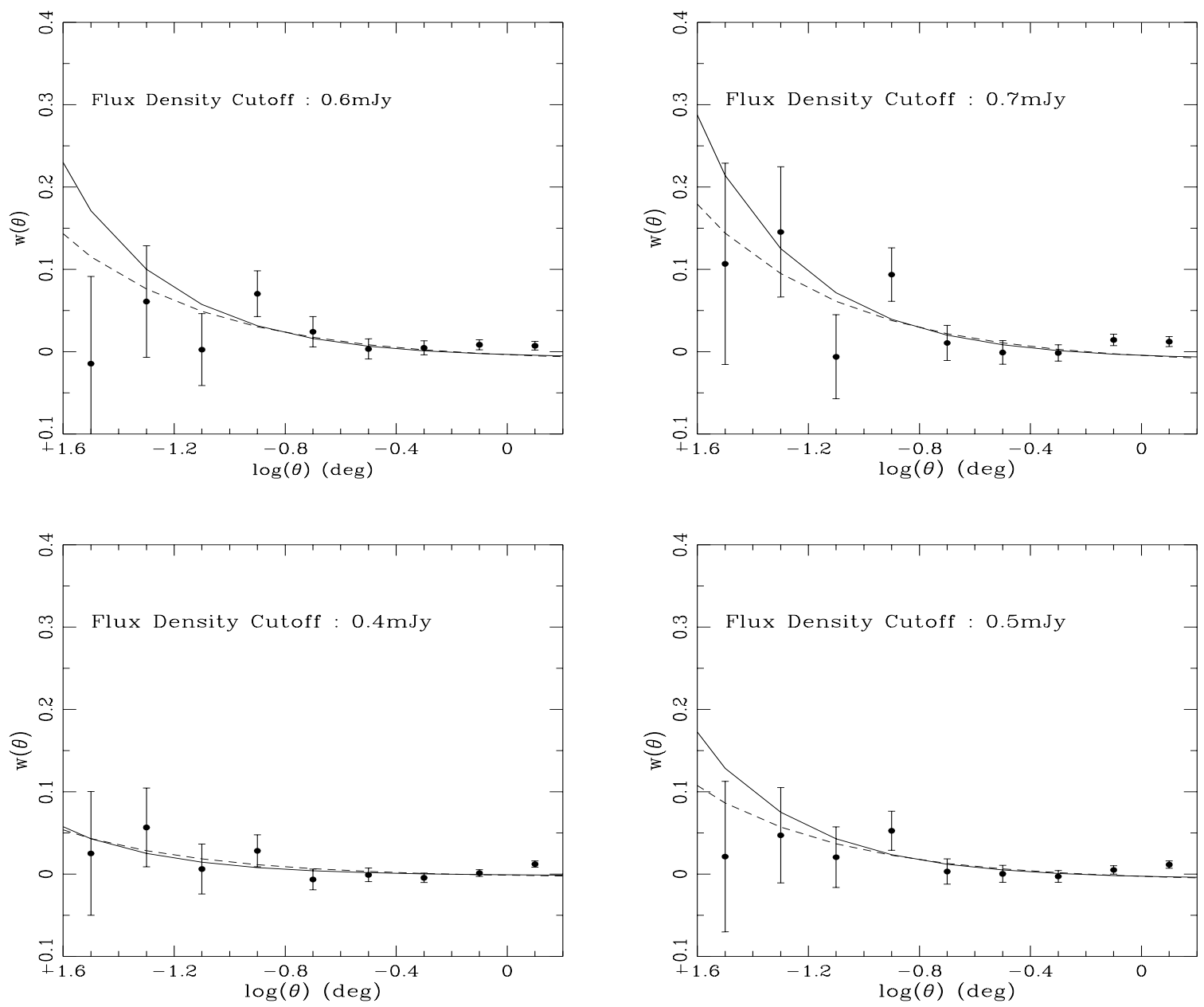

Fig. 3. Angular correlation function $w(\theta)$ of the Phoenix radio survey for subsamples with flux density cutoffs at $0.4,0.5,0.6$ and $0.7 \mathrm{mJy}$. The error bars shown are Poisson estimates. The solid and dashed lines represent the fits to the data assuming $\delta=0.8$ and $\delta=1.1$ respectively. Points in the range $3^{\prime}<\theta<20^{\prime}$ are used in the fitting algorithm
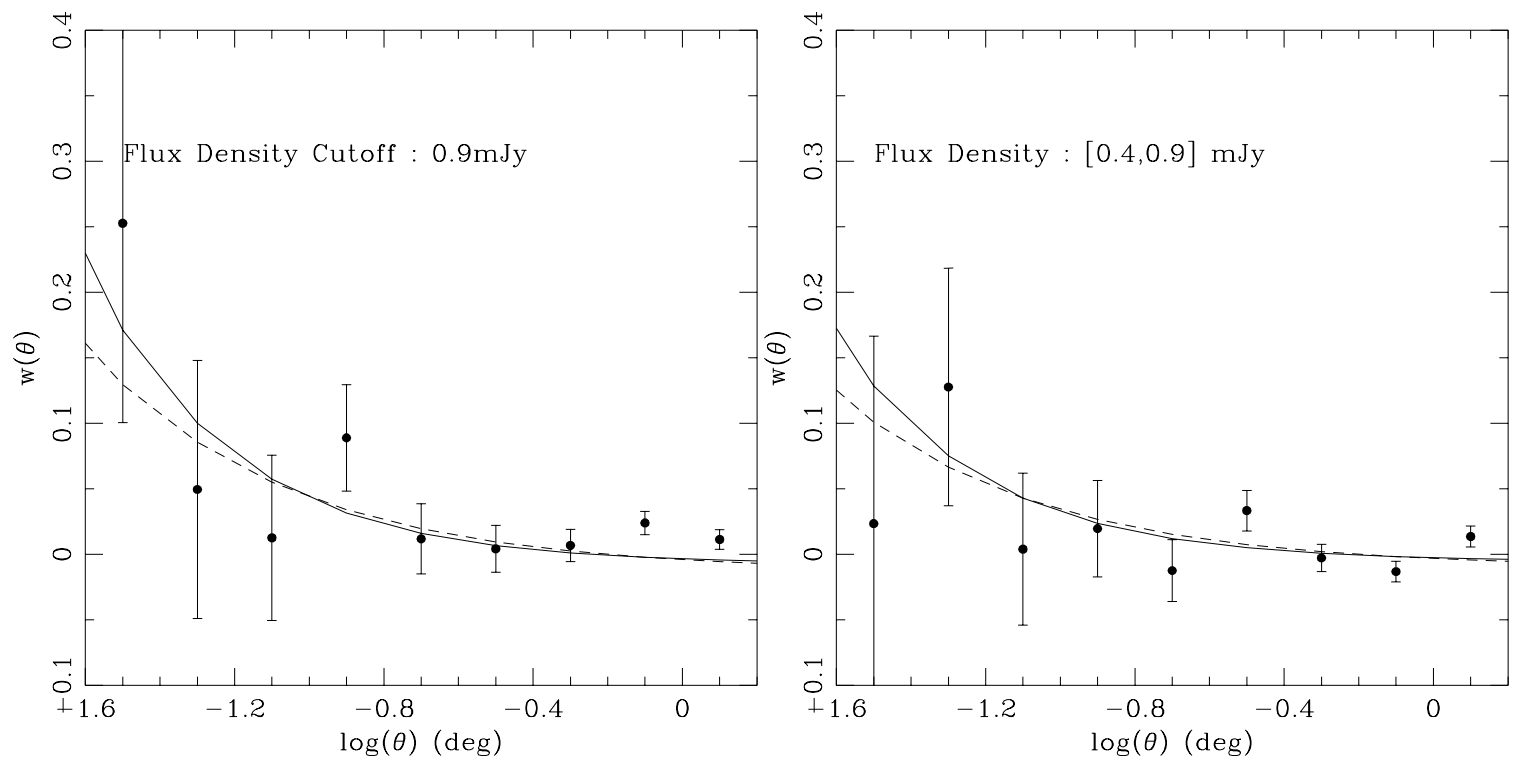

Fig. 4. Angular correlation function for sources with flux densities $S_{1.4}>0.9$ mJy and $0.4<S_{1.4}<0.9$ mJy. The error bars shown are Poisson estimates. The solid and dashed lines represent the fit to the data assuming $\delta=0.8$ and $\delta=1.1$ respectively. Points in the range $3^{\prime}<\theta<20^{\prime}$ are used in the fitting algorithm 


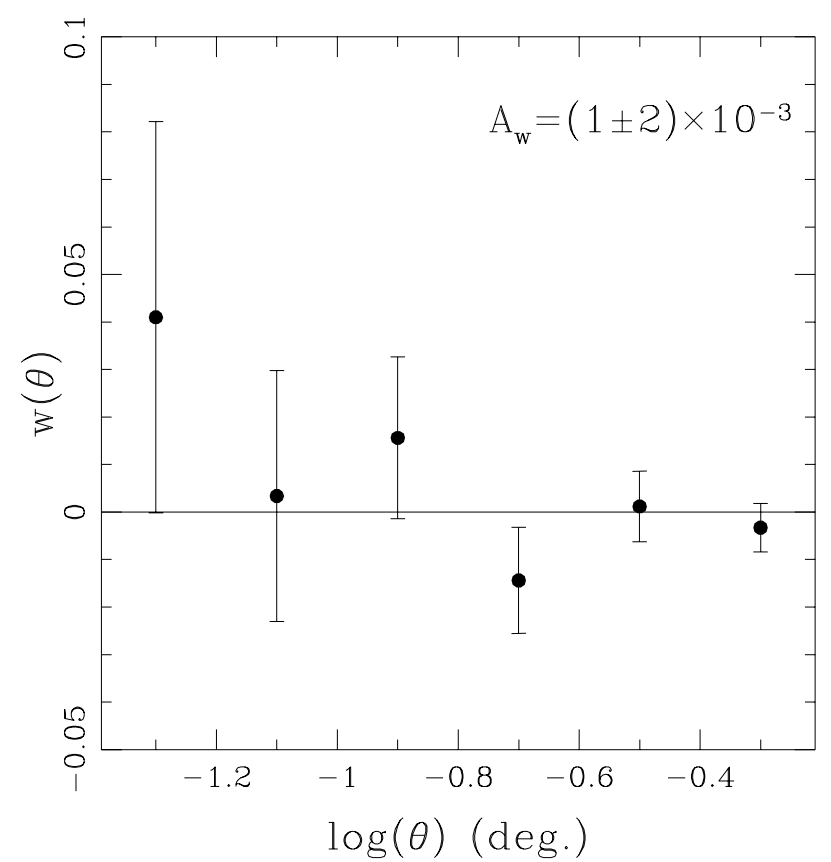

Fig. 5. The angular correlation function for a random sample of points. The error bars are Poisson estimates

and $0.4 \leq S_{1.4} \leq 0.9$ mJy sub-samples. This might indicate a low correlation amplitude at faint flux densities (e.g. $A_{w} \leq 310^{-3}, \gamma=1.8, S_{1.4} \geq 0.4 \mathrm{mJy}$ ), that cannot be detected by the small size of the Phoenix field. However, one should be cautious about this interpretation, since at $0.4 \mathrm{mJy}$ the sample is also likely to be affected by incompleteness.

To further investigate the sensitivity of our results to cosmic variance, artificial galaxy catalogues are constructed with a built-in correlation function of the form $A_{w} \theta^{-\delta}$. Then, we attempt to recover the correlation amplitude from a field having the same geometry as the Phoenix survey.

The algorithm used to generate simulated catalogues with projected hierarchical power-law clustering is described by Infante (1994) and is similar to that employed by Soneira \& Peebles (1978). Firstly, two points separated by an angle $\theta_{1}$ are randomly placed within a $50 \times 50$ degrees area. Each of these points serves as the center of a new pair of randomly oriented points with angular separations $\theta_{2}=\theta_{1} / \lambda$, where $\lambda$ is a real number. The four points generated at the previous step are the new centers for pairs of points separated by $\theta_{3}=\theta_{2} / \lambda$. Therefore the $L$-th step produces $2^{L-1}$ pairs with angular distance $\theta_{L}$ between the points of the pair.

Adopting a value $\lambda=\theta_{l} / \theta_{l+1}=1.8$ for the ratio of separations between successive levels, produces a powerlaw distribution of points with exponent $\delta \approx 0.8$ (Soneira $\&$ Peebles 1978). The angular distance between points at the first level is set $\theta_{1}=1.5 \mathrm{deg}$. Additionally, the number of hierarchical levels, $L$, is drawn from a uniform probabil-

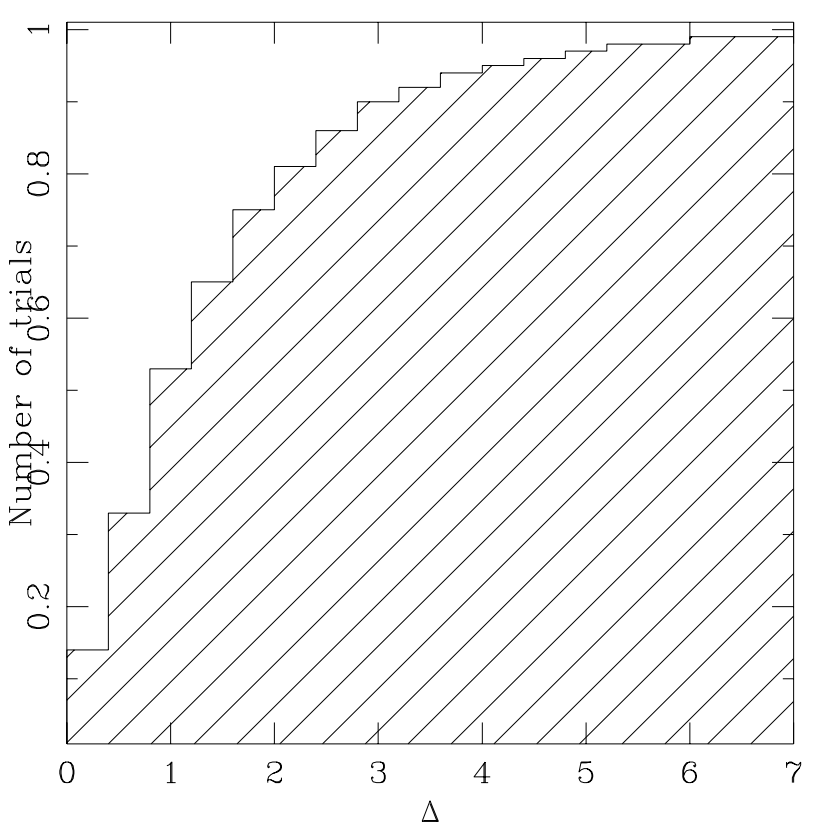

Fig. 7. Cumulative distribution of $\Delta$ defined in Eq. (11)

ity distribution in the interval $\left[L_{\min }, L_{\max }\right]=[6,9]$. The minimum and maximum number of levels are such that the smallest angular separation of the points of a pair is less than $\approx 1$ arcmin. However, these parameters produce too many points at each level, resulting in large angular correlation amplitudes, $A_{w}$, compared to those found in bright radio samples (Loan et al. 1997; Cress et al. 1996). Therefore, each point is assigned a fractional survival probability, $f$. This step does not change the form of the built-in angular correlation function (Bernstein 1994), but allows tuning of $A_{w}$. Values of $f$ between 0.01 and 0.05 produce amplitudes in the range $110^{-3}$ to $2010^{-3}$ respectively. Here we only consider amplitudes in the interval $110^{-3}<A_{w}<1010^{-3}$, similar to those found in brighter radio samples (Cress et al. 1996). The parameters used for four such simulations (trials $A-D$ ) are listed in Table 3. The estimated $w(\theta)$ for trials $B, D$ are plotted in Fig. 6.

The procedure described above is repeated until the surface density of points within the $50 \times 50$ degrees area exceeds the surface density of sources of the present radio sample, at a given flux density cutoff. The angular correlation function is then calculated from the central $36 \mathrm{deg}^{2}$ region, to ensure a uniform surface density of points. The amplitude of the correlation function is estimated by fitting the following function to the observations

$w(\theta)=A_{w}^{\operatorname{sim}} \times \theta^{-0.8}-\omega_{\Omega}$,

where the integral constraint, $\omega_{\Omega}$, is $0.54 A_{w}^{\operatorname{sim}}$ for $\delta=$ -0.8 . The fitting is performed for angular separations between 3 to 50 arcmin. Additionally, for a given mock catalogue, the angular correlation function is also calculated for nine fields, distributed within the central region of the $50 \times 50$ degree area, each having the geometry of the 

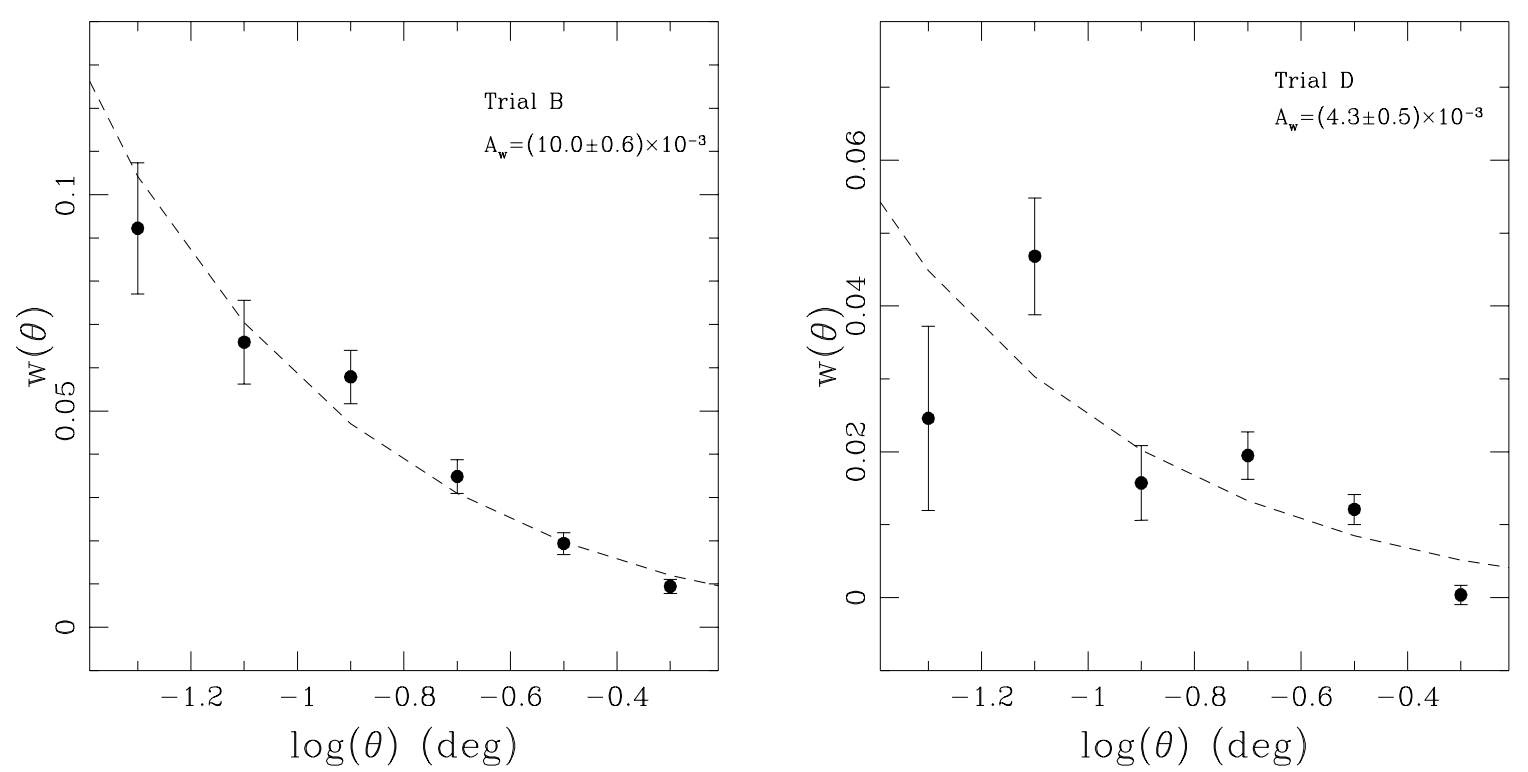

Fig. 6. The angular correlation function for the simulations $B$ and $D$ calculated from an area $\approx 10$ times larger than that of the Phoenix field. The error bars are Poisson estimates

Table 3. Parameters used to construct simulated catalogues. The densities of 145, 170 and 200 sources per $\mathrm{deg}^{2}$, correspond to the surface densities of the present sample at flux density cutoffs of $0.6,0.5$ and $0.4 \mathrm{mJy}$ respectively

\begin{tabular}{cccccc}
\hline Trial & $\lambda$ & $L_{\min }$ & $L_{\max }$ & $f$ & density $\left(\mathrm{deg}^{-2}\right)$ \\
\hline$A$ & 1.8 & 6 & 9 & 0.03 & 145 \\
$B$ & 1.8 & 6 & 9 & 0.04 & 170 \\
$C$ & 1.8 & 6 & 9 & 0.01 & 170 \\
$D$ & 1.8 & 6 & 9 & 0.02 & 200 \\
\hline
\end{tabular}

Phoenix survey. The amplitude, $A_{w}^{\text {est }}$, and the Poissonian uncertainty, $\sigma_{\text {Poisson, }}$, are calculated (see Sect. 3) for each of these smaller fields and then compared to $A_{w}^{\text {sim }}$. The difference, $\Delta$, between $A_{w}^{\text {est }}$ and $A_{w}^{\text {sim }}$ normalised to the Poisson standard deviation is defined

$\Delta=\frac{\left|A_{w}^{\mathrm{sim}}-A_{w}^{\mathrm{est}}\right|}{\sigma_{\text {Poisson }}}$.

For a given survival probability, $f$, and surface density of objects (corresponding to a flux density cutoff) six artificial catalogues are generated. The cumulative distribution of $\Delta$ values (Fig. 7 ), shows that in $\approx 70 \%$ of the trials, $A_{w}^{\text {est }}$ lies within $\approx 2 \sigma_{\text {Poisson }}$ from $A_{w}^{\text {sim }}$. For a Gaussian distribution, this is the definition of one standard deviation. Therefore, the simulations carried out here indicate that the angular correlation amplitudes determined from a radio survey with similar characteristics (i.e. geometry, surface density of objects) to those of the Phoenix survey, are representative of the faint radio population amplitudes within two Poisson standard deviations. This error is smaller than that estimated by the bootstrap resampling technique $\left(\approx 3 \sigma_{\text {Poisson }}\right.$; see Sect. 3$)$, suggesting that cosmic variance is not significantly affecting the present study. However, it is likely that the simulations presented here do not correctly model the higher order correlations produced by gravitational instabilities, on which the $w(\theta)$ errors depend. Nevertheless, the Poisson errors estimated here are independent of higher order correlations and are only meaningful when compared relative to the Poisson uncertainties of the real data-set. In the rest of this study we retain the bootstrap uncertainties as the formal errors, since this method has been widely used to assess the accuracy of $w(\theta)$.

\section{The model}

Let us assume that the evolution of clustering of galaxies with redshift is parametrised by $\epsilon$ such that their spatial correlation function is (Phillipps et al. 1978)

$\xi(r, z)=\left(\frac{r}{r_{0}}\right)^{-\gamma}(1+z)^{-(3+\epsilon)}$,

where $r$ is the proper length and $r_{0}$ the correlation length for $z=0$, corresponding to the scale where the clustering becomes non-linear. The clustering evolution parameter has the property that if $\epsilon \geq 0$ the physical length of a typical cluster reduces with time, resulting in a growth of clustering in proper coordinates. Values of $\epsilon<0$ result in reduction in clustering strength with time. The case of $\epsilon \approx-1.2$ corresponds to clusters of fixed size in co-moving coordinates.

If the redshift distribution $\mathrm{d} N / \mathrm{d} z$ of galaxies is known, then the angular correlation function $w(\theta)$ can be related to $\xi(r)$ by the Limber equation (Limber 1953; Phillipps et al. 1978)

$w(\theta)=C r_{0}^{1-\gamma} \theta^{-(\gamma-1)} \int_{0}^{\infty} D(z)^{1-\gamma} g(z)^{-1}$ 


$$
\begin{gathered}
\times(1+z)^{-(3+\epsilon)}\left(\frac{\mathrm{d} N}{\mathrm{~d} z}\right)^{2} \mathrm{~d} z \\
\times\left[\int_{0}^{\infty} \frac{\mathrm{d} N}{\mathrm{~d} z} \mathrm{~d} z\right]^{-2} .
\end{gathered}
$$

Here, $D(z), g(z)$ and $C$ are defined as

$$
\begin{aligned}
& D(z)=\frac{c}{H_{\mathrm{o}} q_{\mathrm{o}}^{2}} \frac{q_{\mathrm{o}} z+\left(q_{\mathrm{o}}-1\right)\left(\sqrt{1+2 q_{\mathrm{o}} z}-1\right)}{(1+z)^{2}}, \\
& g(z)=\frac{c}{H_{\mathrm{o}}} \frac{1}{(1+z)^{2} \sqrt{1+2 q_{\mathrm{o}} z}}, \\
& C=\sqrt{\pi} \frac{\Gamma((\gamma-1) / 2)}{\Gamma(\gamma / 2)} .
\end{aligned}
$$

It is evident that the exponent $\gamma$ of the spatial correlation function is related to the index $\delta$ of the angular correlation function via the relation $\gamma=\delta+1$.

Unlike optically selected galaxies, the redshift information of the faint radio population is sparse and restricted only to sources with optical counterparts that are relatively bright. Therefore, to predict the amplitudes of the correlation function using Eq. (13) for different input parameters $\left(\epsilon, r_{0}, \gamma\right)$, we need to use a model radio luminosity function (RLF) to estimate their redshift distribution. In the present work the evolving radio luminosity functions of Dunlop \& Peacock (1990) at 2.7 GHz (Model 1) and Rowan-Robinson et al. (1993) at $1.4 \mathrm{GHz}$ (Model 2), extrapolated to faint flux densities, are used.

Dunlop \& Peacock (1990) considered the free form luminosity function of both flat and steep spectrum radio galaxies. Their proposed evolutionary model implies that the radio sources of higher power evolve faster, with the evolution of flat and steep spectrum radio sources being similar. Here we employ their RLF1 model (using their RLF2, RLF3 and RLF4 models give similar results) shifted to $1.4 \mathrm{GHz}$ and converted to $\mathrm{WHz}^{-1}$. The transformation from $2.7 \mathrm{GHz}$ to $1.4 \mathrm{GHz}$ was carried out assuming a power law spectral index $\alpha\left(S_{\nu} \propto \nu^{-\alpha}\right)$ of 0.8 for steep spectrum and 0.0 for flat spectrum radio sources. The redshift distributions at different flux limits for Dunlop \& Peacock models are shown in Fig. 8. The median of the redshift distribution for flux limits of $0.1,0.5$ and $1 \mathrm{mJy}$ is $0.84,0.85$ and 0.91 respectively.

Rowan-Robinson et al. (1993) divided the radio population into spirals, which they showed to be indistinguishable from the starburst galaxies detected at $60 \mu \mathrm{m}$ by IRAS, and ellipticals. For the former population we employ Saunders et al. (1990) "warm" IRAS galaxy component luminosity function at $60 \mu \mathrm{m}$ translated to $1.4 \mathrm{GHz}$ using the empirical relation derived by Helou et al. (1985). For ellipticals, following Rowan-Robinson et al. (1993), we use Dunlop \& Peacock (1990) pure luminosity evolution model RLF6 (parameters from their Table C3). To match the source counts and the redshift distribution of the optically brighter radio sources, pure luminosity evolution for the spiral galaxy component is invoked as described in
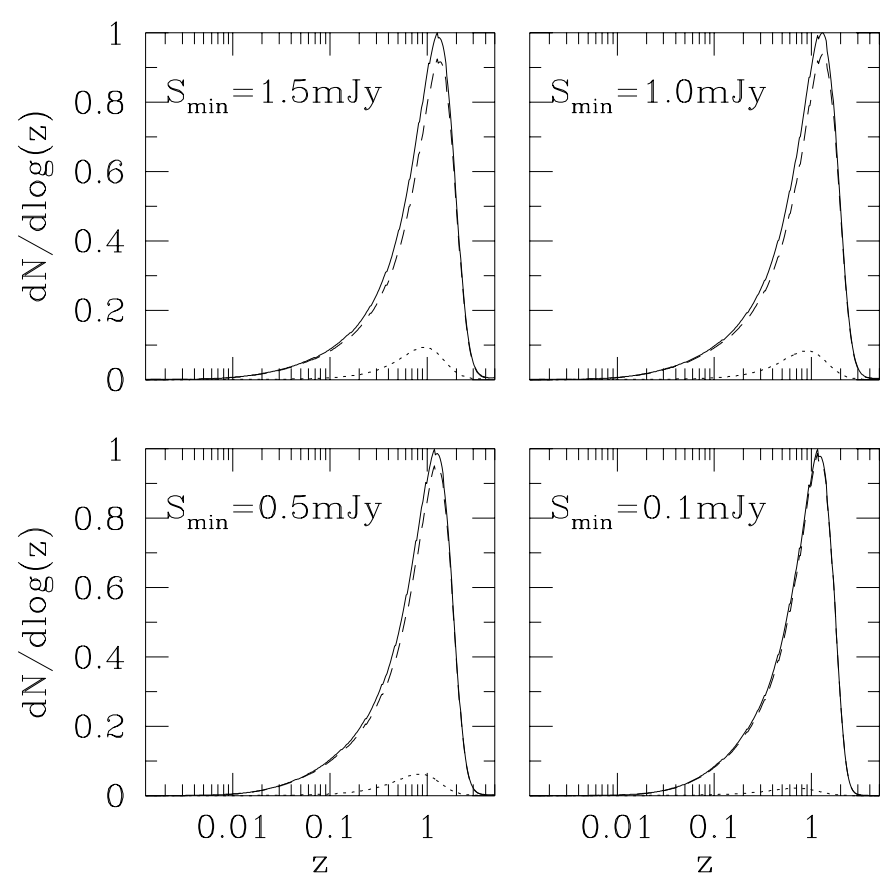

Fig. 8. Redshift distributions for different flux density cutoffs using the radio luminosity function introduced by Dunlop \& Peacock (1990). Dashed line: Steep spectrum radio sources; Dotted line: Flat spectrum radio sources ; Solid line: both populations. The normalisation of the redshift distribution is arbitrary

Rowan-Robinson et al. (1993) and Hopkins et al. (1998). The predicted redshift distributions at different flux density limits are plotted in Fig. 9. The median of the redshift distribution for flux limits of $0.1,0.5$ and $1 \mathrm{mJy}$ is $1.34,1.37$ and 1.44 respectively, significantly larger than the median values predicted by the model developed by Dunlop \& Peacock (1990). Thus, the correlation amplitudes predicted by this RLF model are lower compared to the previous one, for the same parameters $r_{0}, \epsilon$.

Using Eq. (13) we predict the amplitudes of $w(\theta)$ for different values of $\epsilon, r_{0}, \gamma$ and for different radio luminosity functions. The value of $\gamma$ is fixed to either 1.8 or 2.1 , with the models calculated for each value of $\gamma$ independently. Upper limits for the clustering length $r_{0}$ for $\epsilon=-1.2$, are listed in Table 4 . The error estimates of $r_{0}$ are calculated from the $3 \sigma_{\text {Poisson }}$ correlation amplitude uncertainties (Table 2).

\section{Discussion}

Peacock \& Nicholson (1991) have shown that apparently bright radio sources $(S>0.5 \mathrm{Jy})$ in the redshift range $0.01<z<0.1$ are spatially correlated, following a powerlaw behaviour $\xi=\left(r / 11 h^{-1} \mathrm{Mpc}\right)^{-1.8}$. Loan et al. (1997), using two large-area $4.85 \mathrm{GHz}$ radio surveys covering $70 \%$ of the sky, conclude that the $2-\mathrm{D}$ distribution of radio sources brighter than $50 \mathrm{mJy}$ is consistent with a 

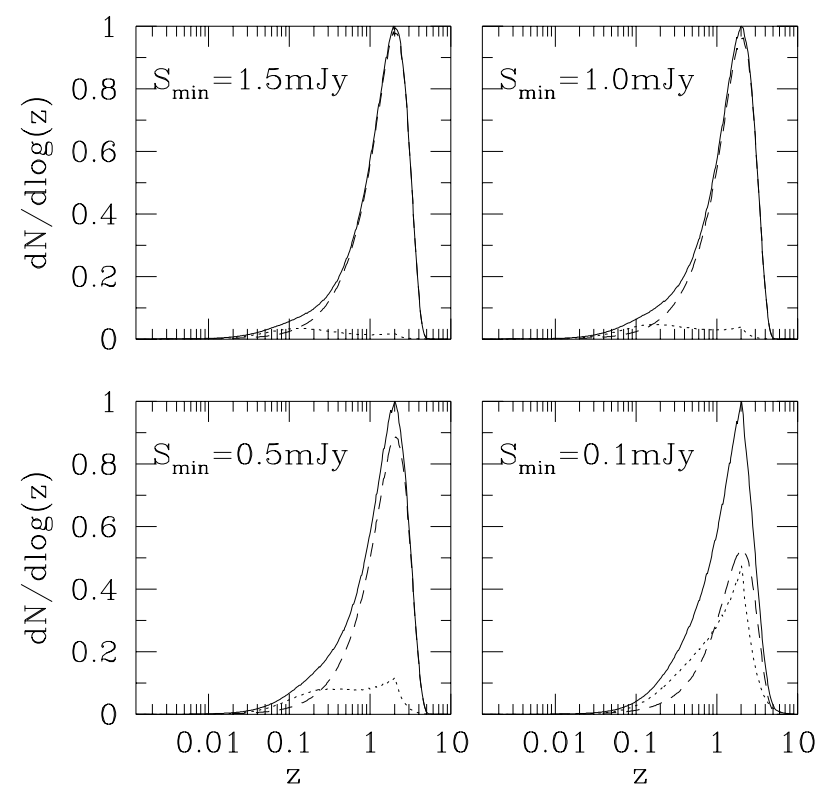

Fig. 9. Redshift distributions for different flux density cutoffs using the radio luminosity function described by RowanRobinson et al. (1993). Dashed line: Ellipticals; Dotted line: Spirals; Solid line: both populations. The normalization of the redshift distribution is arbitrary

Table 4. Upper limits of the clustering length, for $\epsilon=-1.2$ and for two RLF models. Model 1: Dunlop \& Peacock (1990) RLF; model 2: RLF described by Rowan-Robinson et al. (1993). The superscripts are the upper limits to the errors in the $r_{0}$ estimates, corresponding to the bootstrap uncertainties of the angular correlation amplitude

\begin{tabular}{ccccc}
\hline & $r_{0}^{\gamma=(2.1)} h^{-1} \mathrm{Mpc}$ & \multicolumn{2}{c}{$r_{0}^{\gamma=(1.8)} h^{-1} \mathrm{Mpc}$} \\
\hline$S_{1.4}(\mathrm{mJy})$ & model 1 & model 2 & model 1 & model 2 \\
\hline$>0.4$ & $4^{+3}$ & $5^{+3}$ & $6^{+6}$ & $7^{+9}$ \\
$>0.5$ & $7^{+2}$ & $9^{+3}$ & $9^{+6}$ & $11^{+9}$ \\
$>0.9$ & $8^{+4}$ & $10^{+5}$ & $11^{+8}$ & $13^{+10}$ \\
{$[0.4,0.9]$} & $7^{+8}$ & $9^{+6}$ & $9^{+12}$ & $12^{+12}$ \\
\hline
\end{tabular}

correlation length in the range $13<r_{0}<18 h^{-1} \mathrm{Mpc}$ and an evolution parameter $-1.2<\epsilon<0$. This result is based on the RLF models developed by Dunlop \& Peacock (1990) and a value of $\gamma=1.8$. In a separate study, Cress et al. (1996) estimated the angular correlation function of the FIRST radio survey for sources with flux density $S_{1.4}>1$ mJy. Assuming $\epsilon=-1.0$ and $\gamma=2.1$, they found $r_{0}=6-8 h^{-1} \mathrm{Mpc}$ (Cress et al. 1997).

The $r_{0}$ upper limits estimated in this study at different flux density cutoffs agree with the estimates derived from brighter radio samples (Cress et al. 1997; Loan et al. 1997). Because of the small number of sources in each of the independent flux-limited sub-samples $(\approx 318)$ the uncertainties are large and thus we cannot conclude from the present sample if there is a change in the clustering properties of radio sources with flux density.

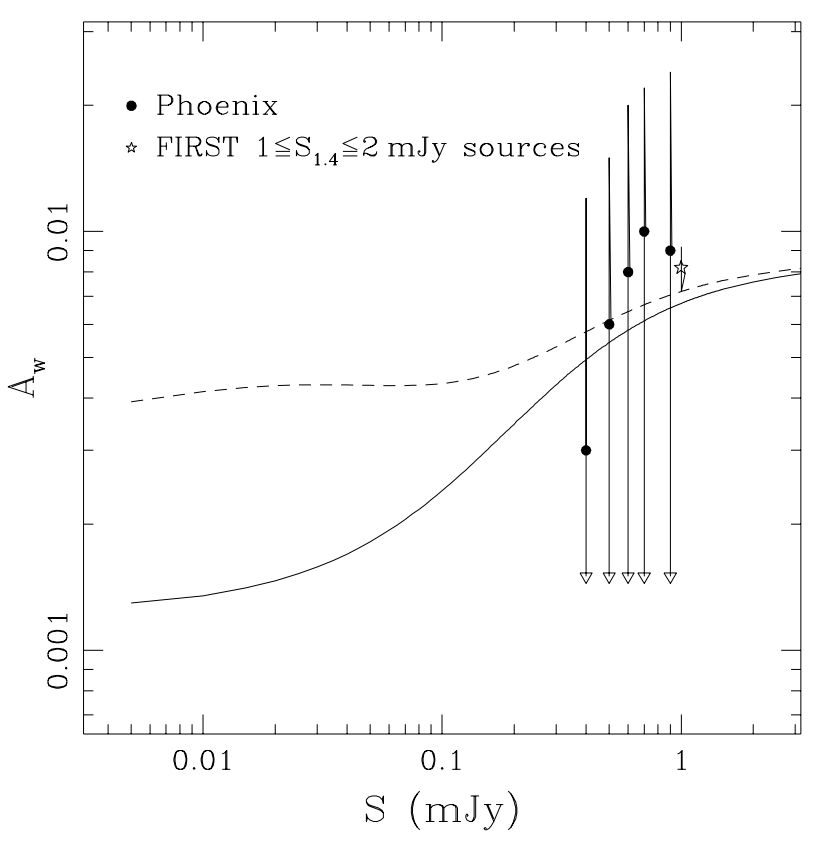

Fig. 10. Predicted amplitude of the angular correlation function at different flux density cutoffs, assuming that the radio population consists of starbursts and elliptical galaxies with different clustering properties Solid line: $r_{0}=5 h^{-1} \mathrm{Mpc}$ for starbursts, $r_{0}=11 h^{-1} \mathrm{Mpc}$ for ellipticals (Model $A$ ); Dashed line: $r_{0}=11 h^{-1} \mathrm{Mpc}$ for both populations (Model $B$ ). The clustering evolution parameter is taken to be $\epsilon=-1.2$ and $\gamma=1.8$ for both populations. Also shown are the estimated amplitudes from this work (filled circles; upper limits) and for sources with $1 \leq S_{1.4} \leq 2$ mJy detected in the FIRST survey

Figure 10 compares the correlation amplitude upper limits from this study with those calculated for the radio sources detected in the FIRST radio survey with $1 \leq S_{1.4} \leq 2 \mathrm{mJy}$ (Cress et al. 1996). The agreement is good, even within the Poisson errors.

In recent years, deep radio surveys, (e.g. Windhorst et al. 1985; Fomalont et al. 1997; Hopkins et al. 1998) have shown a flattening in the slope of the normalised number counts at sub-mJy levels, revealing an excess of faint radio sources over the "normal" radio population of giant ellipticals and QSOs. To address this problem, models invoking strong evolution of either spiral galaxies (Condon 1989) or star-forming IRAS galaxies (Rowan-Robinson et al. 1993; Hopkins et al. 1998) have been combined with RLF models of the "normal" radio population. This scenario is supported by photometric and spectroscopic studies revealing that the sub-mJy radio sources can be identified with galaxies exhibiting evidence of increased star formation (Benn et al. 1993; Windhorst et al. 1985). Furthermore, studies of local galaxies with enhanced star formation (late spirals, IRAS galaxies, HII galaxies), has shown that these objects are more weakly clustered $\left(r_{0} \approx\right.$ $2-4 h^{-1} \mathrm{Mpc}$, for $\gamma \approx 1.5-1.7$; Davis \& Geller 1976 ; Giovanelli et al. 1986; Saunders et al. 1992) than E/S0 galaxies. This implies that the sub-mJy population could 
be more weakly clustered than the E/S0 objects that host the majority of apparently brighter radio sources. Support for this view was advanced by Cress et al. (1996) who found that the slope of the angular correlation function of the sources with $1<S_{1.4}<2 \mathrm{mJy}$ is flatter compared to that found for $S_{1.4}>3 \mathrm{mJy}$. They interpret this result as being due to the increased contribution from starburst galaxies at lower flux density limits, which have flatter angular correlation functions compared to ellipticals, which dominate at brighter flux densities. A similar argument is used by Peacock (1997) to explain the apparent conflict between the value of $r_{0}=6.5 h^{-1} \mathrm{Mpc}$ found for a sample of radio sources brighter than $2.5 \mathrm{mJy}$ and the value $r_{0}=11 h^{-1} \mathrm{Mpc}$ found by Peacock \& Nicholson (1991) for sources brighter than $500 \mathrm{mJy}$ and redshifts in the range $0.01<z<0.1$.

To explore further the implications of the two scales of clustering, and in particular to explore the potential to eliminate competing models by observations, the amplitude of the angular correlation function was estimated, by adopting a simple model (Model $A$ ) in which the radio population consists of two components, one dominating at brighter ( $>1 \mathrm{mJy}$ ) fluxes, with correlation length $r_{0}=11 h^{-1} \mathrm{Mpc}(\gamma=1.8)$ and the other dominating at sub-mJy levels with $r_{0}=5 h^{-1} \mathrm{Mpc}(\gamma=1.8)$ similar to that found for local starburst galaxies. Any crosscorrelation between the two radio populations is ignored. The clustering evolution parameter is taken to be $\epsilon=-1.2$ and our RLF model 2 is employed to predict the redshift distribution of the two radio populations at faint flux densities. In Fig. 10 we plot the flux density cutoff against the amplitude of the angular correlation function calculated from model $A$. For comparison, the expected relation assuming the same value of $r_{0}=11 h^{-1} \mathrm{Mpc}(\gamma=1.8)$ for the two populations (Model $B$ ), is also plotted, along with the upper limits for the angular correlation amplitudes, $A_{w}$, calculated in the present study for different flux density cutoffs. The uncertainties are too large to allow discrimination between the two models.

It is an interesting exercise to predict the depth and the solid angle subtended by a radio survey that would reveal at a $3 \sigma$ significance level if the clustering properties of faint radio sources were significantly different from those of the brighter ones as a result of the changing population. This is accomplished by estimating the uncertainty in $A_{w}$ for a survey of a given solid angle and completeness limit, as described in Appendix A. This then is compared to the difference $\Delta A_{w}$ between the correlation amplitudes predicted at the same flux density cutoff from Models $A$ and $B$. To discriminate between the two models at a $3 \sigma$ confidence level, the uncertainty in $A_{w}$ should be 3 times smaller than $\Delta A_{w}$. The results are shown in Fig. 11, where the flux density cutoff is plotted against the uncertainty in $A_{w}$ for different survey areas. The solid line delimits the area in the parameter space in which $\delta A_{w}$ is at least 3 times smaller than $\Delta A_{w}$ and hence defines the locus of

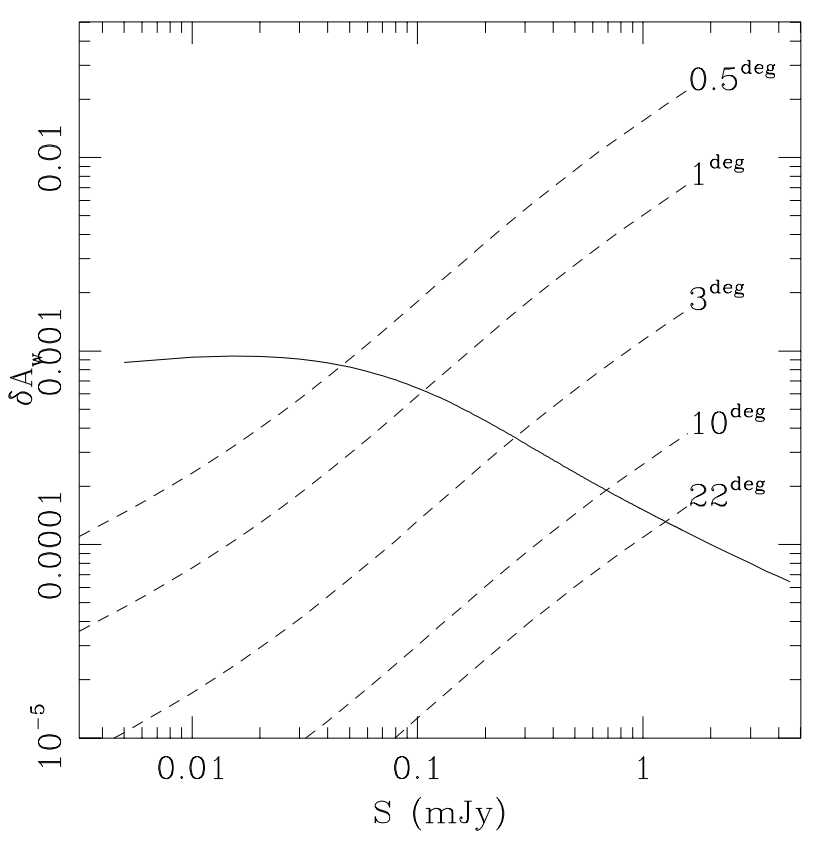

Fig. 11. Flux density cutoff versus the estimated uncertainty in the amplitude of the angular correlation function. Dashed lines correspond to surveys of circular geometry with different radii. The Phoenix survey has a radius of $1^{\circ}$, while the FIRST radio survey is subtending, at the moment, an area corresponding to a radius of $\approx 22^{\circ}$. The solid line represents the locus of $3 \sigma$ confidence level discrimination between models $A$ and $B$ described in the text

$3 \sigma$ confidence level discrimination between the simplified models $A$ and $B$. We conclude that either a very deep survey $\left(S_{\mathrm{lim}}=40 \mu \mathrm{Jy}\right)$ over $\approx 1 \mathrm{deg}^{2}$ or a survey over a larger area at a brighter limit (e.g. at $S_{\lim }=0.2 \mathrm{mJy}$ over $\approx 27 \mathrm{deg}^{2}$ ) is required to discriminate between models $A$ and $B$ and thus reveal if there exists a weakly clustered radio population at faint flux densities. Our analysis shows that the FIRST radio survey, $\left(S_{\mathrm{lim}}=1 \mathrm{mJy}\right)$ covering, at the moment, an area of $\approx 1500 \mathrm{deg}^{2}$, is also appropriate for this purpose.

\section{Conclusions}

Using a deep, homogeneous survey covering an area of $\sim 3 \mathrm{deg}^{2}$, we have investigated the clustering properties of faint (sub-mJy) radio sources by calculating the angular correlation function of the sample. Simulations have been carried out to investigate the significance of our results. It has been demonstrated that the amplitudes estimated here imply a non-uniform distribution of radio sources, albeit at the $2 \sigma$ significance level.

Furthermore, we find that the correlation amplitude upper limits estimated here, despite the large uncertainties, are consistent with the results derived in other studies from larger samples at brighter flux density limits. Spectroscopic and photometric studies of sub-mJy radio 
sources show that the majority have properties similar to starburst galaxies. Since local galaxies, with increased star formation, exhibit weaker clustering than average, it is tempting to link the clustering properties of the sub-mJy population with those of local starburst galaxies. However, the present data, due to small number statistics, cannot reveal a change in radio source clustering properties with flux density. Nevertheless, we have demonstrated that future surveys having sensitivities at $\mu \mathrm{Jy}$ flux density levels and covering large areas on the sky, will be suitable to explore possible changes of the correlation amplitude with flux density.

Acknowledgements. AG is supported by a scholarship from the State Scholarships Foundation of Greece (S.S.F.). The work of $\mathrm{LC}$ and $\mathrm{AH}$ is supported by the Australian Research Council and the Science Foundation for Physics within the University of Sydney. We thank the referee for useful comments and suggestions that improved this paper.

\section{Appendix A: Angular correlation function amplitude uncertainty}

The uncertainty in the amplitude of the angular correlation function of a fiducial radio survey with limiting flux density $S_{\text {lim }}$, subtended over a solid angle $\Omega$, is estimated here. To simplify the procedure, the following assumptions are made:

1. the area subtended by the survey has a circular geometry;

2. the angular correlation function of radio sources is a power law of the form $w(\theta)=A_{w} \theta^{-\delta}$, with the index $\delta$ being fixed;

3. the values of $w(\theta)$ at different angular separations are uncorrelated;

4. The uncertainty in $w(\theta)$ follows Poisson statistics (Peebles 1980) and is given by

$$
\begin{aligned}
\delta w(\theta) & =\sqrt{\frac{1+w(\theta)}{D D},} \\
& =\frac{1}{\sqrt{\frac{1}{2} N(N-1)<\mathrm{d} \Omega>/ \Omega}},
\end{aligned}
$$

where DD is the number of data-data pairs with angular separations $\theta$ and $\theta+\mathrm{d} \theta, N$ is the number of sources above the flux density limit and $<\mathrm{d} \Omega>$ is the mean value of solid angle subtended by annuli with inner and outer radii $\theta$ and $\theta+\mathrm{d} \theta$ respectively. The value of $\langle\mathrm{d} \Omega\rangle$ is affected by the field boundaries. In the present study, this is estimated by creating simulated catalogues of sources distributed randomly over the area of the survey and calculating the expected number of random points, RR, with separations $\theta$ to $\theta+\mathrm{d} \theta$. This is given by

$$
R R=\frac{1}{2} N_{R}\left(N_{R}-1\right) \frac{<\mathrm{d} \Omega>}{\Omega},
$$

where $N_{R}$ is the number of sources in the simulated dataset. The assumption of Poisson errors underestimates the uncertainty in $A_{w}$ (Bernstein 1994).

The assumptions (iii) and (iv) allow us to use least squares fitting techniques to estimate the uncertainty in $A_{w}$

$\delta A_{w}=\left(\sum_{i=\theta_{\min }}^{\theta_{\max }}\left(\frac{\theta_{i}^{-\delta}-\omega_{\Omega}}{\delta w\left(\theta_{i}\right)}\right)^{2}\right)^{-\frac{1}{2}}$,

where $\omega_{\Omega}$ is the integral constraint defined in Sect. 3 . The summation is carried out between a minimum $\left(\theta_{\min }\right)$ and a maximum $\left(\theta_{\max }\right)$ angular separation within equally separated $\log$ arithmic bins of width $\Delta \log \theta=0.2(\theta$ in degrees). The former is taken to be $3^{\prime}$ and the latter is set three times smaller than the radius of the survey, while it cannot be larger than $1^{\circ}$. This is because in real surveys one needs to minimise the effects of field boundaries when estimating $A_{w}$. Additionally, one is interested in the value of $A_{w}$ on small scales, while for larger scales the angular correlation function may deviate from a power law. For example in the optical wavelengths Maddox et al. (1990a) found a break in $w(\theta)$ at scales $\theta \approx 1.5^{\prime \prime}$.

\section{References}

Becker R.H., White R.L., Helfand D.J., 1995, ApJ 450, 559

Benn C.R., Wall J.V., 1995, MNRAS 272, 678

Benn C.R., Rowan-Robinson M., McMahon R.G., Broadhurst T.J., Lawrence A., 1993, MNRAS 263, 98

Bernstein G.M., 1994, ApJ 424, 569

Carlberg R.G., Cowie L.L., Songaila A., Hu E., M., 1997, ApJ 484,538

Condon J., 1984, ApJ 287, 461

Condon J.J., 1989, ApJ 338, 13

Cress C.M., Helfand D.J., Becker R.H., Gregg M.D., White L.R., 1996, ApJ 473, 7

Cress C.M., FIRST collaboration, 1997, astro-ph/9711232

Davis M., Geller M.J., 1976, ApJ 208, 13

Davis M., Peebles P.J.E., 1983, ApJ 267, 465

Dunlop J.S., Peacock J.A., 1990, MNRAS 247, 19

Georgakakis A., Mobasher B., Cram L., Hopkins A., Lidman C., Rowan-Robinson M., 1999, MNRAS 306, 708

Giovanelli R., Haynes M.P., Chincarini G.L., 1986, ApJ 300, 77

Fisher B.K., Davis M., Strauss A.M., Yahil A., Huchra J., MNRAS 1994, 266, 50

Fomalont E.B., Kellermann K.I., Richards E.A., Windhorst R.A., Partridge R.B., 1997, ApJ 475, L5

Hamilton A.J.S., 1993, ApJ 417, 19

Helou G., Soifer B.T., Rowan-Robinson M., 1985, ApJ 298, L7

Hewett P.C., 1982, MNRAS 201, 867

Hopkins A., Mobasher B., Cram L., Rowan-Robinson M., 1998, MNRAS 296, 839

Hudon J.D., Lilly J.S., 1996, ApJ 469, 519

Infante L., 1994, A\&A 282, 353

Infante L., Pritchet C.J., 1995, ApJ 439, 565

Kooiman B.L., Burns J.O., Klypin A.A., 1995, ApJ 448, 500

Landy S.D., Szalay S.A., 1993, ApJ 412, 64

Limber D.N., 1953, ApJ 117, 134 
Ling E.N., Frenk C.S., Barrow J.D., 1986, MNRAS 223, 21p

Loan A.J., Wall J.V., Lahav O., 1997, MNRAS 286, 994

Loveday J., Maddox S.J., Efstathiou G., Peterson B.A., 1995, ApJ 442, 457

Maddox S.J., Efstathiou G., Sutherland W.J., Loveday J., 1990a, MNRAS 242, 43

Maddox S.J., Efstathiou G., Sutherland W.J., Loveday J., 1990b, MNRAS 242, 43

Magliocchetti M., Maddox S.J., Lahav O., Wall J.V., 1998, MNRAS 300, 257

Masson C., 1979, MNRAS 188, 261

Mo H.J., Jing Y.P., Borner G., 1992, ApJ 392, 452

Peacock J.A., 1985, MNRAS 217, 601

Peacock J.A., Nicholson D., 1991, MNRAS 253, 307

Peacock J.A., 1997, Proceedings of the KNAW Colloquium, The Most Distant Radio Galaxies, Amsterdam, October 1997, astro-ph/9712068

Peebles P.J., 1980, The Large Scale Structure of the Universe. Princeton: Princeton University Press

Phillipps S., Fong R., Fall R.S., Ellis S.M., Macgillivray H.T., 1978, MNRAS 182, 673

Oort M.J.A., 1987a, Ph.D. Thesis, University of Leiden
Oort M.J.A., 1987b, A\&AS 71, 221

Richards E.A., 1999, astro-ph/9908313

Roche N., Eales S., Hippelein H., 1998a, MNRAS 295, 946

Roche N., Eales S., 1998b, astro-ph/9803331

Rowan-Robinson M., Benn C.R., Lawrence A., McMahon R.G., Broadhurst T.J., 1993, MNRAS 263, 123

Saunders W., Rowan-Robinson M., Lawrence A., et al., 1990, MNRAS 242, 318

Saunders W., Rowan-Robinson M., Lawrence A., 1992, MNRAS 258, 134

Shaver P.A., Pierre M., 1989, A\&A 220, 35

Soneira R.M., Peebles P.J.E., 1978, AJ 83, 845

Villumsen J.V., Freudling W., Da Costa L.N., 1997, ApJ 481, 578

Webster B.L., 1976, MNRAS 175, 61

Windhorst R.A., Miley K.G., Owen F.N., Kron R.G., David C.K., 1985, ApJ 289, 494

Windhorst R.A., Mathis D., Neuschaefer L., 1990, ASP Conf. Ser. 10, 389

Windhorst R.A., Fomalont E.B., Partridge R.B., Lowenthal J.D., 1993, ApJ 405, 498 